\title{
亚稳态球霰石相碳酸钙的调控制备进展
}

\author{
蒋久信 ${ }^{1,2}$, 吴 月 ${ }^{1}$, 何 瑶 $^{1}$, 高 松 ${ }^{1}$, 张 晨 ${ }^{1}$, 沈 粀 $^{3}$, 刘嘉宁 ${ }^{3,4}$
}

(1. 绿色轻质材料与加工湖北工业大学协同创新中心 武汉 $430068 ; 2$. 湖北工业大学材料与化学工程学院武汉 430068; 3. 南京信息工程大学 物理与光电工程学院, 南京 210044; 4. TU. Braunschweig, Institut für Pharmazeutische Technologie, Mendelssohnstr. 1, D-38106, Braunschweig, Germany)

摘 要: 球霰石以其独特的机械、物理和化学性能, 在日用品与生物医学等领域表现出广阔的应用前景。然而在三 种无水碳酸钻晶体中, 球霰石的热力学性能最不稳定, 在后续的反应和处理过程中常常转变为更稳定的文石或方 解石，因此如何抑制球霰石向稳定晶型转变一直都是碳酸钻领域研究的热点。本文在概述了球霰石晶体结构、性质、 应用及其转化途径的基础上，以碳酸钲的三种基本制备体系为线索，综述了碳化法、复分解法、微乳液法和溶剂热 法等传统方法以及自组装单分子膜法、仿生合成法和热分解法等一些新型调控制备球霰石相方法的研究进展，还就 利用添加剂促进球霰石形成与稳定的相关机制加以剖析。文章旨在为球霰石相碳酸钻的有效制备提供理论和实践 的参考。

关 键 词: 球霰石; 碳酸钻; 亚稳相; 调控; 综述

中图分类号: TQ174 文献标识码: A

\section{Progress in Tuning of Metastable Vaterite Calcium Carbonate}

\author{
JIANG Jiu-Xin ${ }^{1,2}$, WU Yue ${ }^{1}$, HE Yao ${ }^{1}$, GAO Song ${ }^{1}$, ZHANG Chen $^{1}$, SHEN Tong ${ }^{3}$, LIU Jia-Ning ${ }^{3,4}$
}

(1. Collaborative Innovation Center of Green Light-Weight Materials and Processing, Hubei University of Technology, Wuhan 430068, China; 2. School of Materials and Chemical Engineering, Hubei University of Technology, Wuhan 430068, China; 3. School of Physics and Optoelectronic Engineering, Nanjing University of Information Science and Technology, Nanjing 210044, China; 4. Institut für Pharmazeutische Technologie, Technische Universität Braunschweig, Mendelssohnstr. 1, D-38106 Braunschweig, Germany)

\begin{abstract}
Vaterite has broad application prospects in the field of daily necessities and biomedical science because of its unique mechanical, physical and chemical properties. However, vaterite is the thermodynamically least stable phase in the three anhydrous crystalline phases of calcium carbonate, and such thermal metastability often transforms it into more stable aragonite or calcite phases, so how to inhibit the phase transformation of vaterite is always a hot topic in the study of calcium carbonate. In this paper, structure, properties, application, and phase transformation from vaterite to aragonite and/or calcite are briefly introduced. Particularly, based on the three basic routes of synthesis of calcium carbonate, some traditional methods, such as carbonization, double decomposition, microemulsion, solvothermal method, and some latest progress, e.g. self-assembly of monolayer, biomimetic synthesis and thermal decomposition methods of tuning and preparation on vaterite are then summarized. Moreover, some analysis on the mechanism of
\end{abstract}

收稿日期: 2016-08-29; 收到修改稿日期：2016-10-29

基金项目：国家自然科学基金(11174075); 湖北省教育厅科学技术研究计划重点项目(D20151405); 绿色轻质材料与加工湖 北工业大学协同创新中心开放基金(201611A02); 校人才启动基金(S8113127001)

National Natural Science Foundation of China (11174075); Science and Technology Research Program of Educational Commission, Hubei Province of China (D20151405); Open Foundation of Collaborative Innovation Center of Green Light-weight Materials and Processing, Hubei Provincial Key Laboratory of Green Materials for Light Industry (201611A02); Initial Funding of Nanjing University of Information Science \& Technology (S8113127001)

作者简介: 蒋久信(1974一), 男，博士，副教授. E-mail: jiuxinjiang@hotmail.com

通讯作者: 刘嘉宁, 教授. E-mail: jianing.liu@tu-braunschweig.de 
formation and stabilization of vaterite by some additives are given. The aim of this review is to provide a more systematic theoretical and practical guidance to fabrication vaterite phase of calcium carbonate.

Key words: vaterite; calcium carbonate; metastable; tuning; review

球霰石(Vaterite)属于无水碳酸钻 $\left(\mathrm{CaCO}_{3}\right)$ 晶体 三种晶型之一, 其热力学稳定性极差, 在一定条件 下, 常常转化为较为稳定的文石(Aragonite)或方解 石(Calcite), 因此通常难以获得。但球霰石以其特有 的结构和性质得到越来越广泛的应用。传统研究基 于球霰石比表面积大、球形分布、分散性好以及双 折射等特有的品质，可以显著提高油墨、塑料、纸张、 涂料等的填充性能和纸张的涂布性质, 改善产品的 光泽度、流动性、物理和印刷性能 ${ }^{[1-3]}$ 。而生物医学 与药物学的最新研究则表明: 球霰石不但可用于制 备羟基磷灰石, 增强骨和牙齿种植体的机械强度 ${ }^{[4]}$, 而且还可促进细胞向造骨细胞发生变异与增殖, 并 随着碳酸根离子 $\left(\mathrm{CO}_{3}{ }^{2-}\right)$ 的释放来中和移植体发炎部 位的 $\mathrm{pH}^{[5]}$ 。另外, 球霰石因其高的孔隙率还可用作 药物载体 ${ }^{[6]}$ 。有效调控亚稳态球霰石相的形成, 不但 为其在传统日用品领域, 而且还为其在生物医学与 药物学等新领域的深入发展和应用奠定了基础。

\section{1 球霰石 $\mathrm{CaCO}_{3}$ 的结构和形貌}

\section{1 球霰石 $\mathrm{CaCO}_{3}$ 的晶体结构}

有关球霰石相 $\mathrm{CaCO}_{3}$ 的晶体结构, 最被广泛认 同的是球霰石具有六方晶体对称 ${ }^{[7-8]}$, 属于 $\mathrm{P}_{3} / \mathrm{mmc}$ 空间群 ${ }^{[9]}$, 其单元的假晶胞参数是 $a^{\prime}=0.413 \mathrm{~nm}$ 和 $c^{\prime}=0.849 \mathrm{~nm}$, 通过比重测量发现, 球霰石晶胞含有 2 个 $\mathrm{CaCO}_{3}$ 分子 $\left(Z^{\prime}=2\right)^{[10]}$ 。在 $X$ 射线衍射 $(X R D)$ 图 谱中, 有 5 个弱的衍射峰不能够被指标化, 是因为 真正的晶胞沿 $c$ 轴旋转 $30^{\circ}$, 所以晶胞参数就变成 $a=\sqrt{3} a^{\prime}=0.716 \mathrm{~nm}, c=2 c^{\prime}=1.698 \mathrm{~nm}, Z=12$, 如图

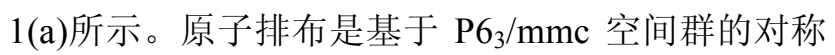

元素构建而成。该模型假定钻原子完全填充，碳酸 根 $1 / 3$ 填充。当碳原子置于棱柱的中心和垂直的碳 酸根做相应排列时，每个氧原子与最近两个钙原子等 距, 结果钙氧原子之间的距离具有预期的数值。在此 基础上, Meyer ${ }^{[11]}$ 提出另外一种结构, 其基本结构单元 在本质上与前一种模型相同, 只是 $\mathrm{CO}_{3}{ }^{2-}$ 的位置对称 不同。在衍射数据中观察到的光谱特征归因于垂直于 $c$ 轴的堆垛层错, 这就导致 $c$ 轴晶胞参数增大 2 或 3 倍, 如图 1(b)所示。

最近的研究认为球霰石的晶体结构是由两种不 同晶体结构结合而成的，即这两种结构共存于一个 假单晶之中, 其中占主导的是六方结构 ${ }^{[12]}$ 。这些不 同的模型都可以从碳酸根离子的不同排列取向、碳 酸根层堆垛次序的多样性和可能的手性形式等方面 解释球霰石的无序。对此, Demichelis 等 ${ }^{[13]}$ 认为球霰 石并非是个单纯的无序结构, 而是由不同结构的复 合体组成, 其结构之间可快速地相互转化, 但每种 结构都有着相似的性质。

\section{2 球霰石 $\mathrm{CaCO}_{3}$ 的颗粒形貌}

颗粒形貌不仅受其内在晶体结构的影响，还会受 到外部制备条件的影响 ${ }^{[14-16]}$ 。球体是球霰石相的典型 形貌。对颗粒表面的研究表明, 球体是由更小的单晶 按照一定的角度排列而成 ${ }^{[17]}$ 。断面形貌表明, 纳米晶 粒发展成定向棒状, 即是从球体中心向四周形成放射 性的纤维状。当纤维数量增大时, 它们末端就会合拢 成一个球体，成为 “球体一哑铃-球体” 的颗粒生长模 式 ${ }^{[15-16,18]}$ 。除了典型的球状结构, 体外合成的球霰石 也有其他复杂形状，如盘状、煎蛋状、棱镜状、各种 分层花状以及由六重对称构成的莲座形结构 ${ }^{[15,19-22]}$ 。
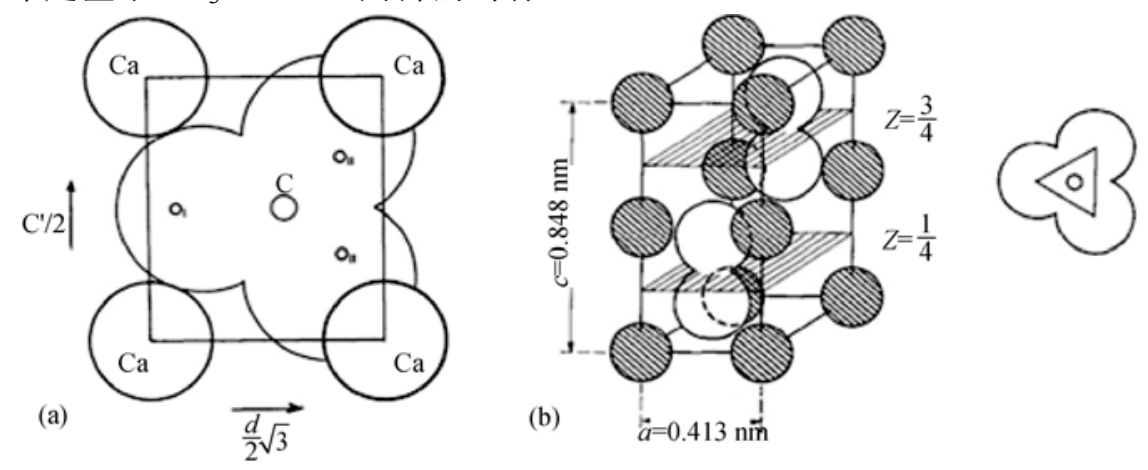

图 1 (a)球霰石中碳酸根和钻原子位置的垂直投影 ${ }^{[10]}$; (b)球霰石的晶胞结构 ${ }^{[11]}$

Fig. 1 (a) Vertical projection of vaterite showing orientation of carbonate group relative to calcium atoms ${ }^{[10]}$;

(b) Structure of the vaterite unit cell ${ }^{[11]}$ 


\section{2 球霰石 $\mathrm{CaCO}_{3}$ 的研究进展}

\section{$2.1 \mathrm{CaCO}_{3}$ 的三种基本制备体系}

一般来说, 液相合成 $\mathrm{CaCO}_{3}$ 可分为三种基本反 应系统: $\mathrm{Ca}(\mathrm{OH})_{2}-\mathrm{H}_{2} \mathrm{O}-\mathrm{CO}_{2}$ 系统、 $\mathrm{Ca}^{2+}-\mathrm{H}_{2} \mathrm{O}-\mathrm{CO}_{3}{ }^{2-}$ 系统和 $\mathrm{Ca}^{2+}-\mathrm{R}-\mathrm{CO}_{3}{ }^{2-}$ 系统。首先, $\mathrm{Ca}(\mathrm{OH})_{2}-\mathrm{H}_{2} \mathrm{O}-\mathrm{CO}_{2}$ 系统(碳化法), 是向 $\mathrm{Ca}(\mathrm{OH})_{2}$ 悬浊液中通入 $\mathrm{CO}_{2}$ 制 备 $\mathrm{CaCO}_{3}$ 的方法。工业上采用石灰石㷽烧、石灰消 化、 $\mathrm{Ca}(\mathrm{OH})_{2}$ 碳化、分离、干燥、分级包装制取纳 米 $\mathrm{CaCO}_{3}$ 。 $\mathrm{Ca}(\mathrm{OH})_{2}-\mathrm{H}_{2} \mathrm{O}-\mathrm{CO}_{2}$ 系统原料丰富、成本 低廉, 具有产品质量好、经济且可生产多种晶形等 优点, 是目前制造纳米级 $\mathrm{CaCO}_{3}$ 主要采用的方法。 其次, $\mathrm{Ca}^{2+}-\mathrm{H}_{2} \mathrm{O}-\mathrm{CO}_{3}{ }^{2-}$ 系统(复分解法), 是将含有 $\mathrm{Ca}^{2+}$ 的盐溶液与含有 $\mathrm{CO}_{3}{ }^{2-}$ 的盐溶液在一定条件下 混合制备纳米 $\mathrm{CaCO}_{3}$ 。该方法通过控制反应物的浓 度、温度及生成物 $\mathrm{CaCO}_{3}$ 的过饱和度, 加入适当的 添加剂等, 可制取各种晶型的 $\mathrm{CaCO}_{3}$ 。但是, 通过 该方法来获取不同晶形的产品成本较高, 一般较少 采用。对于 $\mathrm{Ca}^{2+}-\mathrm{R}-\mathrm{CO}_{3}{ }^{2-}$ 系统( $\mathrm{R}$ 为有机介质), 则是 通过有机介质 $\mathrm{R}$ 来调节 $\mathrm{Ca}^{2+}$ 和 $\mathrm{CO}_{3}{ }^{2-}$ 之间的传质, 从而达到控制晶核生长的目的。依据有机介质 $\mathrm{R}$ 的 不同, 可将该体系分为乳液法和凝胶法, 前者一般 采用液体油为有机介质, 如石蜡油、煤油等; 而后者 则采用有机凝胶, 如有机硅凝胶、琼脂糖凝胶等。

\section{$2.2 \mathrm{CaCO}_{3}$ 各相之间的转化}

$\mathrm{CaCO}_{3}$ 各相之间有着向更稳定结构转化的趋势, 大 致可分为两种途径, 一种是通过溶解再结晶 ${ }^{[23-25]}$ (如图 2
所示), 另一种则是固-固相之间的直接转化 ${ }^{[26]}$ 。第一 种转化途径中, 初始相是无定形碳酸钙(ACC), 其内 部松散, 会快速转变成为球霰石。球霰石有较高的溶 解度, 溶液中球霰石溶解而方解石成核生长。这样 的不断溶解和生长, 使得球霰石向方解石相变。对 于第二种转化途径, 目前研究较少。Politi 等 ${ }^{[26]}$ 发 现 ACC 呈短程有序, 在生物体内可通过固-固相 重排来转化为结晶态。海胆的尖刺是由方解石单 晶组成的光滑且弯曲的一种结构。要形成这种结 构, 首先 ACC 沉积排列成尖刺的形状, 然后再随 时间慢慢实现从 ACC 向球霰石再到方解石单晶的 转变。

\section{3 球霰石 $\mathrm{CaCO}_{3}$ 的调控制备进展}

伴随着对球霰石相结构、相-相转化与稳定机制 等深入理解，一些调控制备球霰石相 $\mathrm{CaCO}_{3}$ 的方法 与技术也逐渐发展并成熟起来, 主要有碳化法、复 分解法、微乳液法和溶剂热法等传统方法以及自组 装单分子膜法、仿生合成法和热分解法等一些新型 方法。

碳化法属于 $\mathrm{Ca}^{2+}-\mathrm{H}_{2} \mathrm{O}-\mathrm{CO}_{2}$ 系统, 是一种最常见 的方法, 其中 $\mathrm{Ca}^{2+}$ 的来源有两种: $\mathrm{Ca}(\mathrm{OH})_{2}$ 悬浊液 和 $\mathrm{CaCl}_{2}$ 溶液。对于 $\mathrm{Ca}(\mathrm{OH})_{2}$ 悬浊液的碳化, 只有使 用特殊的添加剂时才能部分合成出球霰石, 如以 $\mathrm{Ca}(\mathrm{OH})_{2}$ 为钙源, 在碳化体系中添加马来酸䣶 $(\mathrm{MAH})^{[27]}$ 和多糖 ${ }^{[28]}$ 等。相比前者, $\mathrm{CaCl}_{2}$ 溶液的碳化 产物更容易出现球霰石, 其中添加剂仍是影响产物 晶型的重要因素。如图 3 所示, Zhang 等 ${ }^{[29]}$ 利用碳酸 氢铵分解产生的 $\mathrm{CO}_{2}$ 对 $\mathrm{CaCl}_{2}$ 溶液进行碳化, 结果

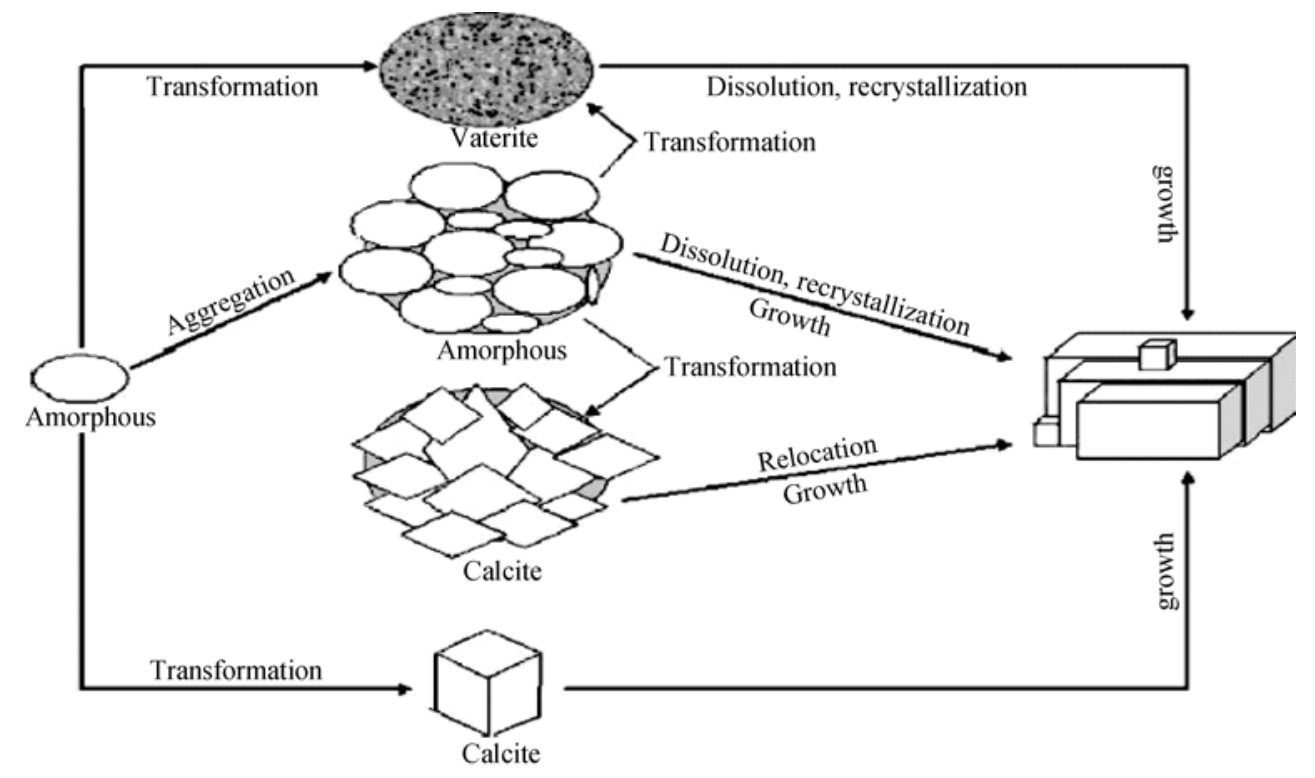

图 $2 \mathrm{CaCO}_{3}$ 从非晶相转变为球霰石球晶和典型方解石的形成示意图 ${ }^{[25]}$

Fig. 2 Schematic depiction of the $\mathrm{CaCO}_{3}$ transformation from amorphous phase to vaterite spherulites and typical calcite ${ }^{[25]}$ 

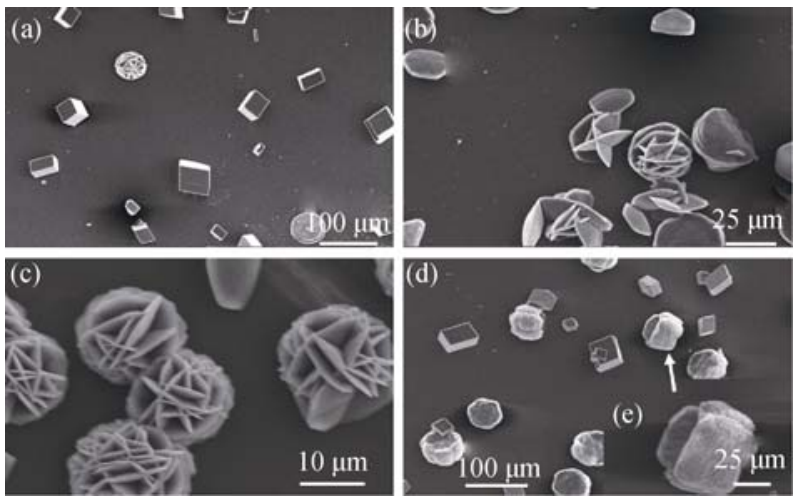

图 3 无水对氨基苯磺酸与 $\mathrm{L}$-赖氨酸不同混合比 $\mathrm{CaCO}_{3}$ 晶体 的 SEM 照片 ${ }^{[29]}$

Fig. 3 SEM images of $\mathrm{CaCO}_{3}$ particles in different concentration ratios between $\mathrm{p}$-aminobenzene sulfonic acid anhydrous and 1-Lys solutions ${ }^{[29]}$

(a) $0.1 \mathrm{~g} / \mathrm{L}: 0.1 \mathrm{~g} / \mathrm{L}$; (b) $0.1 \mathrm{~g} / \mathrm{L}: 0.3 \mathrm{~g} / \mathrm{L}$; (c) $0.1 \mathrm{~g} / \mathrm{L}: 0.5 \mathrm{~g} / \mathrm{L}$; (d) $0.5 \mathrm{~g} / \mathrm{L}: 0.1 \mathrm{~g} / \mathrm{L}$; (e) High magnification of selective area of (d)

表明: 添加剂的混合比例直接影响球形霰石相的含 量, 当添加剂无水对氨基苯磺酸与 $\mathrm{L}$-赖氨酸的混合 比提高至 $1: 5$ 时, 球霰石在其与方解石的混合相中 占主要成分。

复分解法制备球霰石, 属于 $\mathrm{Ca}^{2+}-\mathrm{H}_{2} \mathrm{O}-\mathrm{CO}_{3}{ }^{2-}$ 系 统, 主要受制于温度、 $\mathrm{pH}$ 、溶剂体系、添加剂和其 他因素(如搅拌、超声波 ${ }^{[30-31]}$ )等, 用于制备多形貌的 球霰石。其中, 所用添加剂主要有 $\mathrm{NH}_{3} / \mathrm{NH}_{4}{ }^{+[19] \text { 、对 }}$ 氨基苯甲酸 $(\mathrm{PABA})^{[32]}$ 、聚苯乙烯磺酸钠 $(\mathrm{PSS})^{[31,33]}$ 、 聚乙烯吡咯烷酮 (PVP) ${ }^{[34]}$ 、十二烷基磺酸钠 $(\mathrm{SDSN})^{[34-35]}$ 、聚乙二醇 $(\mathrm{PEG}) 2000 / 10000^{[35]}$ 、三聚 磷酸钠 $(\mathrm{STP})^{[31]}$ 和羧甲基纤维素钠 $(\mathrm{CMC})^{[31]}$ 等。 $\mathrm{Hu}$ 等 ${ }^{[19]}$ 将碳酸氢铵分解产生的 $\mathrm{NH}_{3}$ 通入复分解体系 中, 发现当 $\mathrm{NH}_{3}$ 的扩散速率较快时, 产物以球霰石 为主; 反应时间从 $1 \mathrm{~h}$ 延长到 $4 \mathrm{~h}$, 球霰石的形貌由 球状变为花状。Ramesh 等 ${ }^{[32]}$ 研究了 PABA、溶剂类 型和 $\mathrm{pH}$ 对 $\mathrm{CaCO}_{3}$ 晶型的影响, 发现不加 $\mathrm{PABA}$ 时, 在水一甲醇混合溶液中, 仅当 $\mathrm{pH}>9$ 时, 才会出现球 霰石。加入 $\mathrm{PABA}$ 后, 在水溶液中, $\mathrm{pH}>7$ 时则是以 球霰石为主; 在水一甲醇的混合溶液中, $\mathrm{pH}<8$ 时, 产 物是球霰石和方解石共存, 而 $\mathrm{pH}>8$ 时, 则是纯的 球霰石。夏宏宇等 ${ }^{[33}$ 则将 PSS 作为添加剂, 分别在 水溶液和二甲亚砜(DMSO)-水溶液体系中, 通过混 合沉积制备出球形和橄榄形的球霰石。邹俭鹏等 ${ }^{[34]}$ 研究表明, 仅添加 SDSN 时, 制备的中空微球均为方 解石型, 而只有同时添加 PVP 和 SDSN 时, 才能得到 方解石和球霰石混合的中空微球, 并且随着 SDSN 浓度/PVP 添加量的增加, 中空微球数目均减少, 实 心微球数目均增多。 $\mathrm{Ji}$ 等 ${ }^{[35]}$ 则发现(见图 4), 在复分
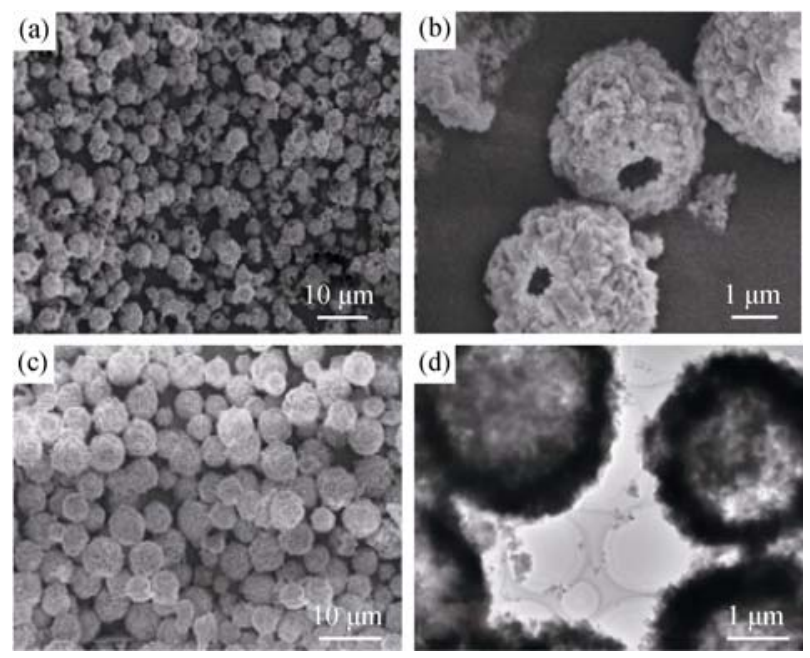

图 4 添加 PEG10000-SDS 的 SEM 和 TEM 照片 ${ }^{[35]}$

Fig. 4 SEM images of $\mathrm{CaCO}_{3}$ hollow spheres obtained in the presence of PEG10000-SDS ${ }^{[35]}$

(a) Low magnification image, (b) high and (c) middle magnification SEM images; and (d) TEM image of $\mathrm{CaCO}_{3}$ hollow spheres obtained in the presence of PEG2000-SDS

解体系中单独添加 PEG2000/10000 或 SDSN, 均得 不到空心微球，而只有同时加入上述添加剂时，才 能得到由方解石和球霰石混合组成的空心微球，同 时添加 PEG10000 和 SDSN 时, 得到表面由菱形结构 组成的空心微球, 而同时添加 PEG2000 和 SDSN 时, 则得到表面更加稳定的空心微球。此外, 汪小红等 ${ }^{[30]}$ 还利用超声波在复分解体系中制备得到荔枝状球霰 石。杨亚因等 ${ }^{[31]}$ 同样利用超声波技术, 发现添加 STP 时，除了在用量极低的条件下 $(\leqslant 0.10 \mathrm{wt} \%)$ 形成的产 物主要为球霰石相外，一般都形成无定形 $\mathrm{CaCO}_{3}$; 添加 CMC 时, 一般都得到方解石相; 而添加 PSS 时, 则得到以球霰石相为主的产物。

微乳液法属于 $\mathrm{Ca}^{2+}-\mathrm{R}-\mathrm{CO}_{3}{ }^{2-}$ 系统, 该方法制备 的 $\mathrm{CaCO}_{3}$ 粒径小且纯度高。其中, 利用微乳液法 ${ }^{[36]}$ 制得的 $\mathrm{CaCO}_{3}$ 晶体中 $90 \%$ 为球霰石相, 粒径均一且 分散性良好。研究还发现, $\mathrm{CaCO}_{3}$ 的粒径随着所加入 油酸与 $\mathrm{CaCO}_{3}$ 质量比的降低而逐渐减小, 当油酸的 加入量为 $2.5 \mathrm{wt} \%$ 时, $\mathrm{CaCO}_{3}$ 平均粒径仅为 $10 \mathrm{~nm}$ 。 而反相微乳液法 ${ }^{[37]}$ 的研究发现, 随着阴离子表面活 性剂十二烷基硫酸钠(SDSN)与水混合比例的提高, 在微乳液中所形成的 $\mathrm{CaCO}_{3}$ 由方解石相明显地转化 成球霰石相。如图 5 所示, Jiang 等 ${ }^{[38]}$ 基于所合成的 $\mathrm{CO}_{2} / \mathrm{N}_{2}$ 可控 $\mathrm{N}^{\prime}$-二十二烷基- $\mathrm{N}, \mathrm{N}-$ 二甲基乙酰胺碳酸 氢( $\mathrm{N}^{\prime}$-dodecyl-N, Ndimethyl acetamidine bicarbonate) 反相微乳液, 成功制备了不同形貌球霰石与方解石 的混合相。 

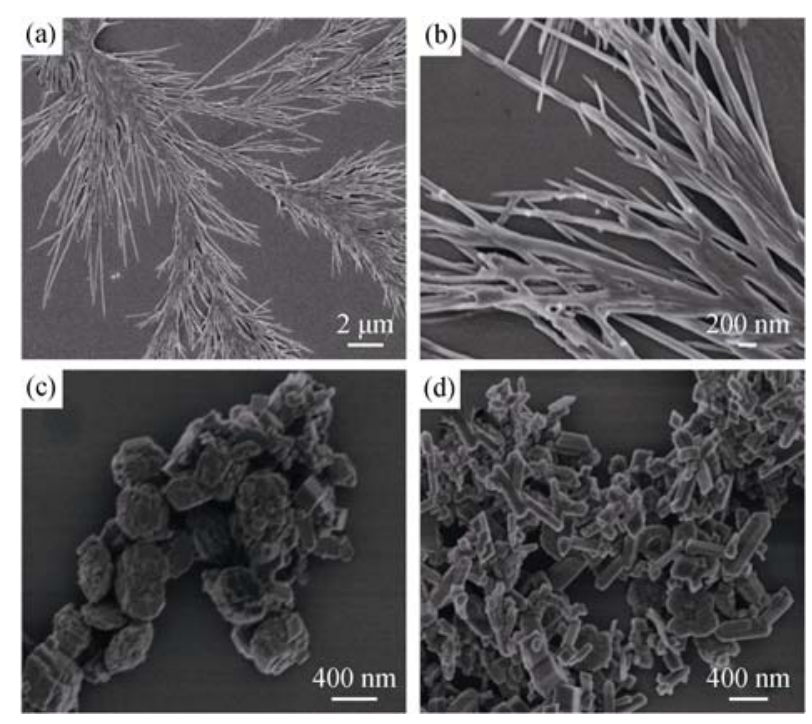

图 5 不同浓度表面活性剂下制备的 $\mathrm{CaCO}_{3}$ 晶体的 $\mathrm{SEM}$ 照片 ${ }^{[38]}$ Fig. 5 SEM images of $\mathrm{CaCO}_{3}$ prepared at different surfactant concentrations ${ }^{[38]}$

(a) $0.1 \mathrm{~mol} / \mathrm{L}$; (b) Magnification of (a); (c) $0.07 \mathrm{~mol} / \mathrm{L}$; (d) $0.04 \mathrm{~mol} / \mathrm{L}$

溶剂热法是通过把一种或几种前驱体溶解在非 水溶剂, 在液相或超临界条件下, 缓慢生成产物的 一种方法。 $\mathrm{Li}$ 等 $^{[7]}$ 在 $100 \sim 150^{\circ} \mathrm{C}$ 范围用溶剂热法得 到球形球霰石相 $\mathrm{CaCO}_{3}$, 发现当溶剂从乙二醇调整 为 1,2 -丙二醇, 再调整到甘油, 温度逐渐升高有利 于生成球霰石。如以乙醇-水为溶剂, 添加适量的羟 基乙叉二膦酸(HEDP), 再采用低温溶剂热法, 可制 备出圆饼状球霰石 ${ }^{[39]}$ 。对采用无添加剂的微波水热 法, 当醋酸钙用量为 $0.9 \mathrm{~g}$, 尿素和醋酸钙配比分别 为 $1.5: 1$ 和 $2: 1$ 时, 可合成方解石和球霰石的混合 相 ${ }^{[40]}$ 。而将微乳液法与溶剂热法结合, 通过对一种 过饱和水包油微乳液体系的被动蒸发, 基于钙离子 与表面活性剂分子中硫酸基的相互作用, 则可获得 一种海绵状的球霰石微球, 如图 6 所示 ${ }^{[41]}$ 。

自组装单分子膜法是制备纳米粒子的一种重要 方法, 主要利用高度有序的单分子膜为基体, 有效 控制在其上成核和生长的纳米粒子的粒径分布、几 何结构和稳定性。An 等 ${ }^{[42]}$ 通过自组装单层膜和多 个可溶性添加剂的相互作用, 发现丝素蛋白和 $\mathrm{Mg}^{2+}$ 可以发挥协同作用(见图 7)。当丝素蛋白用量较低 $(10 \mathrm{~mL})$ 时, 球霰石的含量随 $\mathrm{Mg}^{2+}$ 浓度的增大而增 加; 而当丝素蛋白用量较高 $(20 \mathrm{~mL})$ 时, 随着 $\mathrm{Mg}^{2+}$ 浓度的增大, 产物从无定形的 $\mathrm{CaCO}_{3}$ 变为较稳定的 花状球霰石。其它模板法也可有效地控制 $\mathrm{CaCO}_{3}$ 的 形貌、结构和粒径的重要途径, 常见的有琥珀酸二 (2-乙基己酯)磺酸钠 $(\mathrm{AOT})^{[43]}$ 、多糖(如壳聚糖 ${ }^{[44]}$ )、
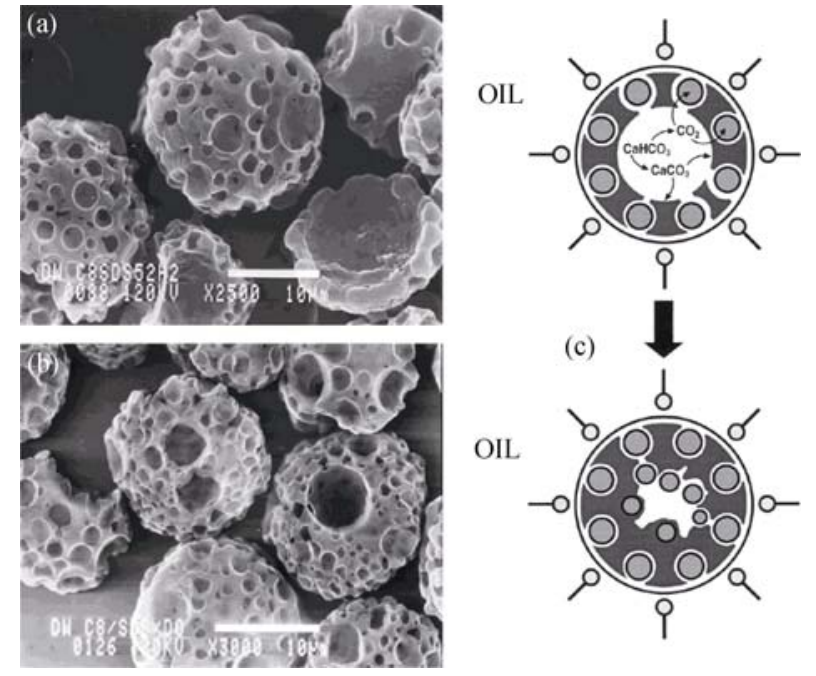

图 6 蒸发水包油饱和微乳液制备海绵状球霰石的扫描电镜 照片 ${ }^{[41]}$

Fig. 6 SEM images showing sponge-like vaterite spheroids prepared by evaporation ${ }^{[4]]}$ from water-in-oil supersaturated microemulsions with compositions of (a) octane : SDS : $\mathrm{CaHCO}_{3}=71: 4: 25(\mathrm{wt} \%) ;$ (b) octane $:$ dodecanol $:$ SDS : $\mathrm{CaHCO}_{3}=70.8: 0.7: 3.5: 25(\mathrm{wt} \%)$; (c) schematic diagram showing the mechanism for the formation of vaterite microsponges in water-in-oil microemulsions

微生物(如真菌、放射菌 ${ }^{[45]}$ )和气泡/乳液 ${ }^{[46]}$ 等。但是, 同样存在模板法不利于球霰石形成的报道, 如采用 碳酸铵分解法, 在乙醇-水体系中, 在 $\mathrm{L}$-半胱氨酸 单分子膜上获得取向为(104)晶面的方解石, 而无自 组装单分子膜的作用, 产物才以球霰石为主 ${ }^{[47]}$ 。

仿生合成法则是通过模拟生物体内的反应和 天然物的结构来合成的一项新型制备技术，其发 展前景广阔。目前 $\mathrm{CaCO}_{3}$ 仿生合成研究的方法主 要分为两大类, 即生物体直接提取有机质和人工 合成具有特殊官能团有机高分子, 前者如恶臭假 单胞菌 ${ }^{[48]}$ 和大豆胰岛素 ${ }^{[49]}$ 等, 后者包括富含羧基 的多肽基双亲水杂化共聚物 ${ }^{[50]}$ 、氨基酸(如 L-缅氨 酸 $^{[51]}$ 、精氨酸 ${ }^{[51]} 、 \mathrm{~L}-$ 丝氨酸 ${ }^{[51]}$ 和 $\mathrm{L}$-赖氨 酸 $^{[52]}$ )、 $\mathrm{PSS}^{[52-53]} 、 \mathrm{~N}$ - 琥珀酰基-O-羟丙基磺酸壳聚糖 $(\mathrm{SA}-\mathrm{HPSCS})^{[54]}$ 、聚( $\mathrm{N}$ - 异丙基丙烯酰胺) $-\mathrm{b}$ - 聚( $(\mathrm{L}-$ 谷氨酸)(PNIPAM-b- PLGA $)^{[55]}$ 、柠檬酸钠 $(S C)^{[56]}$ 、 十二烷磺基苯磺酸钠 $(\mathrm{SDBS})^{[56]}$ 和聚氧乙烯脱水山梨 醇单油酸酯(Tween-80) $)^{[57]}$ 等。如图 8 所示 ${ }^{[51]}$, 以 L-顺 氨酸、L-精氨酸为有机基质分别生成立方方解石和球 形球霰石, 而以 $\mathrm{L}-$ 丝氨酸为有机基质则形成立方方解 石和球形球霰石的混合相。在体系中单独加入 PSS 或 L-赖氨酸, 形成实心球形球霰石; 而同时加入 PSS 和 $\mathrm{L}$-赖氨酸, 在产物中则会出现球霰石微环 ${ }^{[52]}$ 。 

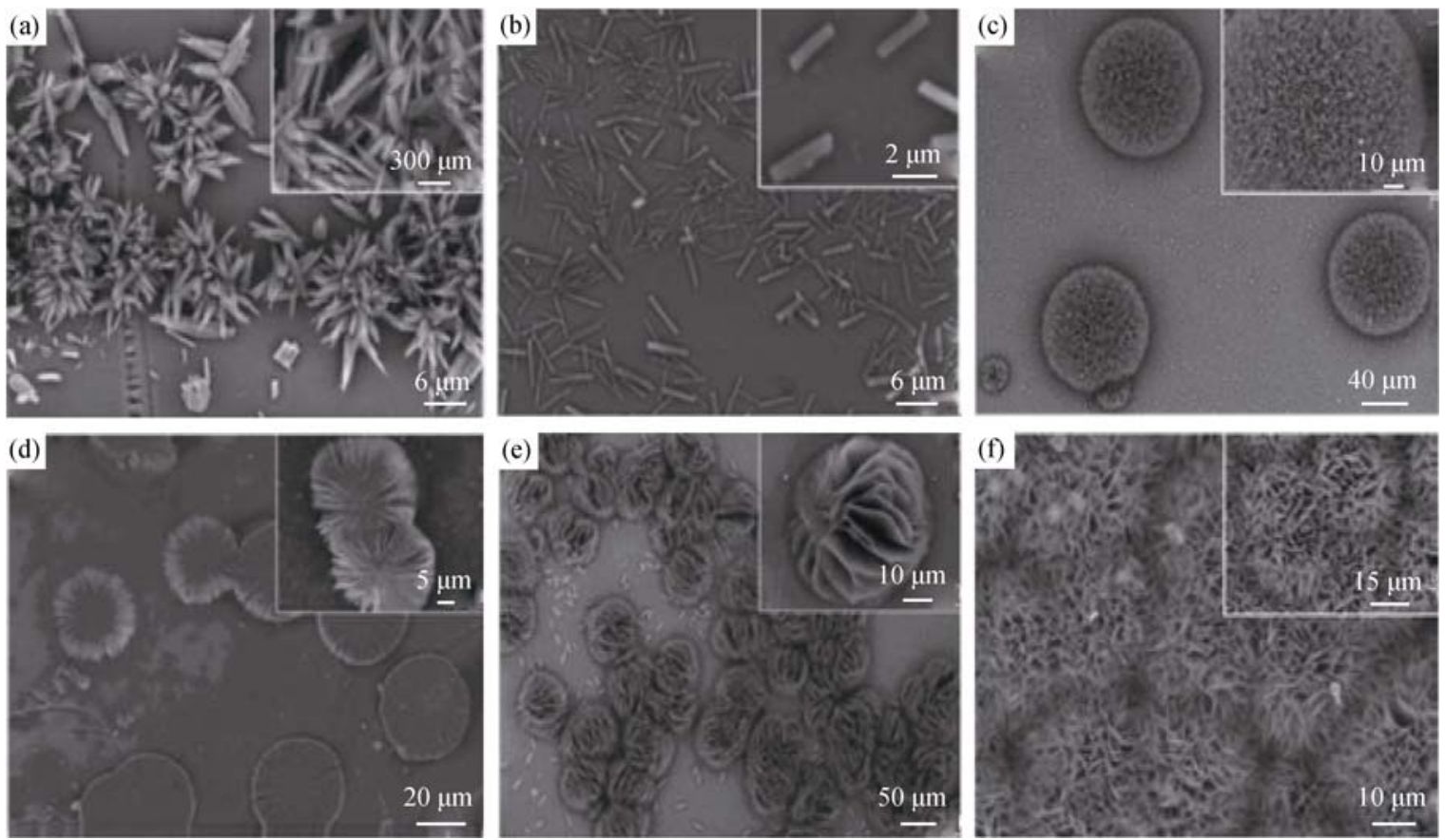

图 $7 \mathrm{CaCO}_{3}$ 的 $\mathrm{SEM}$ 照片，其中 $(\mathrm{a} \sim \mathrm{c})$ 和(d f) 分别为加入 $10 \mathrm{~mL} 、 20 \mathrm{~mL}$ 的丝素蛋白，且所加镁离子浓度( $\mathrm{mmol} / \mathrm{L}$ )分别为(a) 10; (b) 20 ; (c) 50 ; (d) 10 ; (e) 20 ; (f) $50^{[42]}$

Fig. 7 SEM images of $\mathrm{CaCO}_{3}$ where (a-c) and (d-f) with $10 \mathrm{~mL}$ and $20 \mathrm{~mL}$ of silk fibroin, respectively, affter being added $\mathrm{Mg}^{2+}$ at the concentration of (a) 10; (b) 20; (c) 50 ; (d) 10 ; (e) 20 ; (f) $50 \mathrm{mmol} / \mathrm{L}^{[42]}$
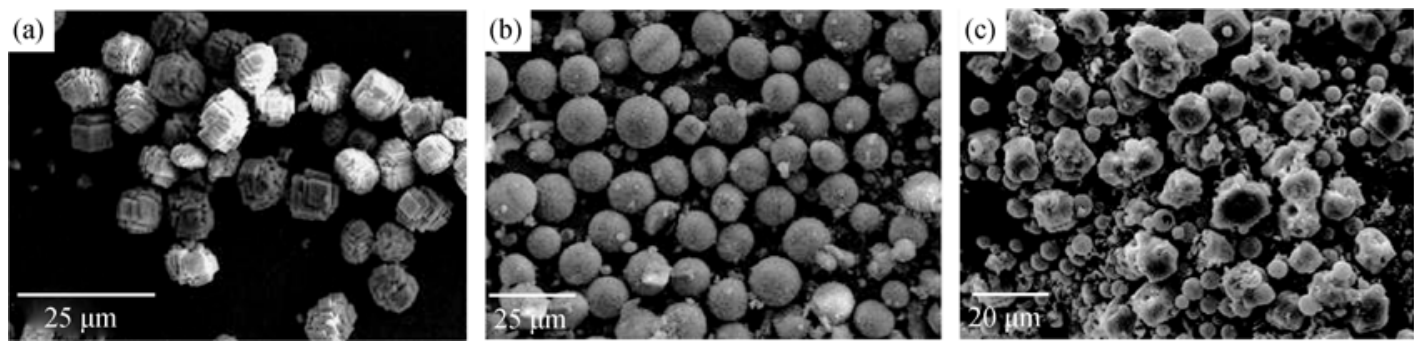

图 8 不同水溶液中制备的 $\mathrm{CaCO}_{3}$ 的 SEM 照片 ${ }^{[51]}$

Fig. 8 SEM images of the $\mathrm{CaCO}_{3}$ prepared in different aqueous solutions ${ }^{[51]}$

(a) L-valine; (b) L-arginine; (c) L-serine

此外, 碳酸氢钙热分解也是一种制备球霰石相 $\mathrm{CaCO}_{3}$ 的新型方法。对此, 我们首先利用碳酸氢钙 饱和溶液热分解制备 $\mathrm{CaCO}_{3}$ 粉体, 分别从分解温度 与分解时间 ${ }^{[58]}$ 、机械摚拌 ${ }^{[59]}$ 等角度控制 $\mathrm{CaCO}_{3}$ 晶相 的组成和颗粒形貌。如图 9 和 10 所示, 当分解温度 为 $70^{\circ} \mathrm{C}$ 时获得大约 $37.4 \mathrm{wt} \%$ 的球霰石相, 但随着温 度的升高, 方解石含量增加, 而球霰石相和文石相 的含量都有所降低。在热分解体系中我们还发现, 大量雪花状、树叶状和花状的超结构亚稳相球霰石 的形成需要在静置状态下来实现, 施加搅拌不利于 球霰石的生成。对此我们从原子堆积的角度分析了 这种微观作用机制(如图 11 所示) ${ }^{[59]}$ 。最近, 对于碳 酸氢钙热分解体系, Zeng 等 ${ }^{[60]}$ 还发现, 加入油酸也 有利于球霰石的生成。

\section{3 促进球霰石相形成与稳定的相关机制}

研究表明，高饱和盐溶液、碱性条件、控制温 度及加入添加剂等能够促使球霰石颗粒形成并使 之稳定存在，其中加入添加剂是最常用的方法。一 般来说, 添加剂稳定球霰石相的作用主要表现在 两个方面: 一是通过与离子的相互作用降低反应前 体的浓度进而降低成核的热力学驱动力; 二是被 吸附在球霰石特定晶面上延迟晶相的成核或阻止 其进一步向稳定晶型的生长 ${ }^{[61]}$ 。一些无机添加剂和 有机质均可促进球霰石的沉淀，包括含氮化合物、 蛋白质、双亲嵌段共聚物、树枝状大分子、聚合物、 醇以及微生物等。具体来说, 主要体现在以下几个 方面。 


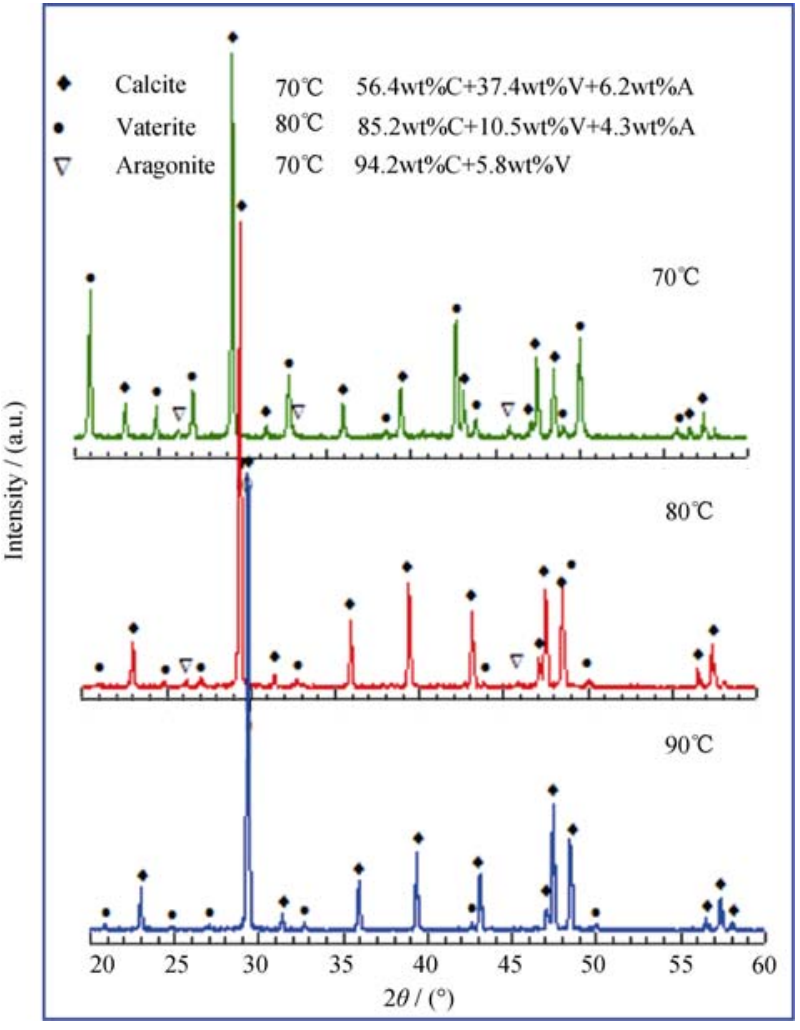

图 9 不同分解温度下碳酸钙的 XRD 图谱 ${ }^{[58]}$

Fig. 9 XRD patterns of $\mathrm{CaCO}_{3}$ decomposed under different temperatures ${ }^{[58]}$

(a) $70{ }^{\circ} \mathrm{C}$; (b) $80{ }^{\circ} \mathrm{C}$; (c) $90{ }^{\circ} \mathrm{C}$

利用氨基酸(如赖氨酸、丙氨酸、亮氨酸)可以促 进和稳定球霰石相 ${ }^{[22]}$ 。通常认为, $\mathrm{C}=\mathrm{O}$ 键的负电荷向 $\mathrm{O}$ 原子移动形成强电场, 该电场对 $\mathrm{Ca}^{2+}$ 有强烈的吸 引作用。依靠静电作用临时固定在 $\mathrm{C}=\mathrm{O}$ 基团附近的 $\mathrm{Ca}^{2+}$ 引发球霰石临界晶核的形成。生物实验表明酰胺 基通过 N-H---O 氢键和球霰石表面的紧密结合能够 控制 $\mathrm{CaCO}_{3}$ 的晶体类型并可以保持球霰石的长时间 稳定 ${ }^{[62]}$ 。

类似地，一些蛋白质已经被用来控制 $\mathrm{CaCO}_{3}$ 的 晶体类型和颗粒形貌, 结果表明蛋白质对结晶的方 向和晶体生长有强烈的影响。鸡蛋中的卵清蛋白可 以通过蛋白质边缘的羧化物捕获 $\mathrm{Ca}^{2+}$, 从而导致
$\mathrm{Ca}^{2+}$ 的富集。而且一旦球霰石晶体形成, 卵清蛋白会 吸附在其表面, 从而阻止其向稳定相的转变 ${ }^{[63]}$ 。

双亲嵌段共聚物(DHBCs)包含两个亲水性的结 构单元，它对 $\mathrm{CaCO}_{3}$ 晶型和形貌的调控可以用 “责 任共担” 来解释：一 $\mathrm{COOH}$ 功能段与纳米晶体或晶 核发生相互作用，另一段通过空间位阻的稳定作用 使大晶粒溶解。两个任务互不干扰，两个段可以独 立地优化晶体类型、颗粒大小、粉体功能和结构 ${ }^{[16]}$ 。 研究表明，中等浓度的聚乙烯亚胺-聚乙酸(PEI-PA) 共聚物功能段是促进小尺寸纯球霰石相晶体生成非 常有效的模板 ${ }^{[16]}$ 。

形成球霰石相的引发核是由于树形分子球形的 三维形貌和高度功能化的表面 ${ }^{[28,64]}$, 普遍应用的聚 酰胺-胺(PAMAM) 有足够多的胺分支可以作为阳离 子的络合位置 ${ }^{[28]}$ 。另一个树形分子一聚丙烯亚胺, 与阳离子型表面活性剂一起作为优先吸引碳酸根离 子的模板形成一个稳定的表面, 为球霰石相的形成 吸附更多的离子 ${ }^{[64]}$ 。

多元醇的羟基 $(-\mathrm{OH})$ 基团可以促进纯球霰石 相的生成 ${ }^{[7-8,65]}$ 。一 $\mathrm{OH}$ 基团形成的强电场吸引晶核 改变球霰石相的表面能并使其在热力学上比文石和 方解石更稳定 ${ }^{[7]}$ 。研究表明, 除了乙醇胺, 添加只具 有一个一 $\mathrm{OH}$ 基团的醇都得不到纯球霰石相 ${ }^{[7,66]}$ 。多 元醇延长了亚稳相的转变时间, 降低了稳定相的生 长速率 ${ }^{[8,65]}$, 从而阻止了向稳定相的再结晶。

一些细菌可以生成 $\mathrm{CO}_{2}$ 和 $\mathrm{NH}_{3}$, 并把 $\mathrm{NH}_{3}$ 转化 成 $\mathrm{NH}_{4}{ }^{+}$和 $\mathrm{OH}^{-}$, 这样使细菌处于碱性环境中。而在 碱性环境使 $\mathrm{CO}_{3}{ }^{2-}$ 的超饱和度增大, 这有助于球霰 石沉淀。因此, 球霰石相容易在细胞周围的微环境 中生成, 或直接在细菌的表面生成。另外, 细菌的细 胞壁含众多表面功能基团，比如羧基、羟基和磷酸 基团, 这些位置都会促进球霰石的结晶并对其起稳 定作用。研究结果还表明, 不同类型的细菌(如土壤 细菌和放射菌)对球霰石沉淀的调节并不显示菌株 特异性 ${ }^{[45,62]}$ 。
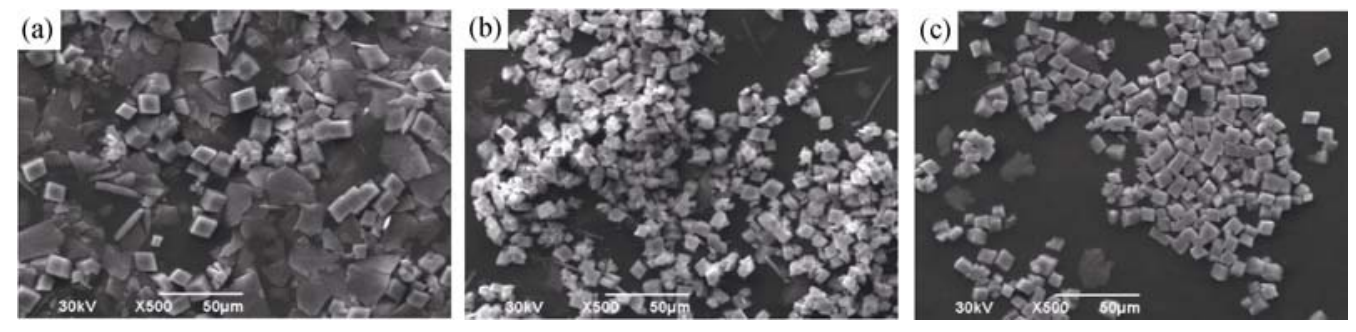

图 10 不同分解温度下 $\mathrm{CaCO}_{3}$ 的 SEM 照片 ${ }^{[58]}$

Fig. 10 SEM images of $\mathrm{CaCO}_{3}$ decomposed under different temperatures ${ }^{[58]}$

(a) $70^{\circ} \mathrm{C}$; (b) $80^{\circ} \mathrm{C}$; (c) $90^{\circ} \mathrm{C}$ 


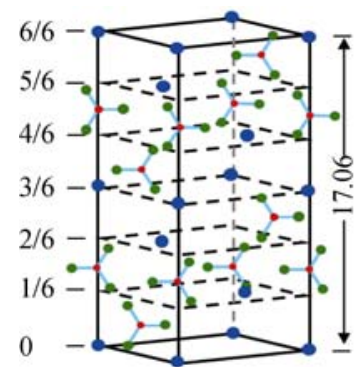

(a)

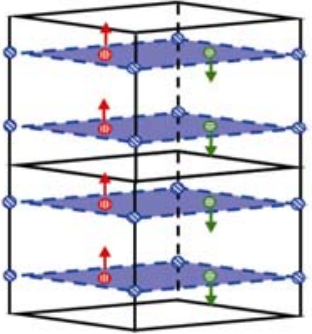

(c)

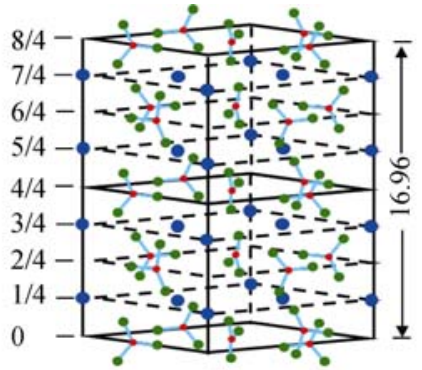

(b)

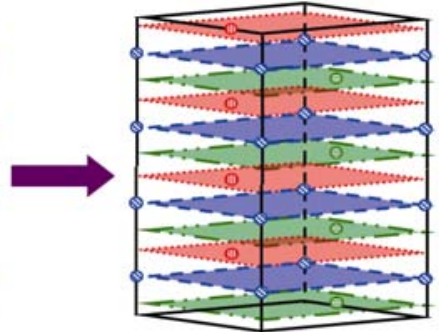

(d)
图 11 (a)和(b)方解石和球霰石的原子结构, (c)球霰石结构中 (0001) 晶面 $\mathrm{Ca}^{2+}$ 的排列以及在摚拌影响下的移动方向, (d) 移 动后 $\mathrm{Ca}^{2+}$ 的排列及移动过程 ${ }^{[59]}$

Fig. 11 Atomic structure of (a) calcite and (b) vaterite, (c) $\mathrm{Ca}^{2+}$ ions stacking in vaterite and the motion direction in the (0001) faces under agitation, and (d) $\mathrm{Ca}^{2+}$ ions stacking in the (0001) faces after motion ${ }^{[59]}$

(i Moving up one third of interplanar crystal spacing; 自 Moving up one third of interplanar crystal spacing; No moving)

\section{4 结束语}

球霰石相的极不稳定性使其需要特殊的制备与 调控技术, 其中高饱和度、温度和 $\mathrm{pH}$ 、静置的热分 解体系等均有利于亚稳相球霰石相的生成。而含有 羧基、磷酸盐、磺酸盐、羟基、羧酸盐和氨基的添 加剂对球霰石相的生成则具有一定的促进作用。同 时, 在合成过程中改变添加剂的浓度、分子量和功 能基团的数量还可以调节颗粒大小和表面形貌、晶 体习性、稳定性以及晶体的生长速率。

目前, 球霰石相 $\mathrm{CaCO}_{3}$ 基于其独特的物理化学 性能已被广泛应用在日用品、生物医学、制药和食 品等众多领域。其中, 最为新颖和应用发展最快的是 其作为层-层自组装的药物载体。研究球霰石相的结 构与形貌形成的调控机制有望提高其对药物的承载 率, 同时调控球霰石晶体的结构与尺寸也可增加细 胞对药物载体的摄取速度。尽管近十年来球霰石相 结构的形成机制与调控技术的研究已有很大发展, 不同形状球霰石颗粒的生长机制、最终结构和公共 特性仍然需要进一步探索。

\section{参考文献:}

[1] CUI Z, CUI C, ZHU Y, et al. Multiple phase inversion of emulsions stabilized by in situ surface activation of $\mathrm{CaCO}_{3}$ nanoparticles via adsorption of fatty acids. Langmuir, 2011, 28(1): 314-320.

[2] LIU S. Stabilized Vaterite. U.S. Patent Application, US20030717 310. 2003.11.19.

[3] DEVENNEY M, FERNANDEZ M, MORGAN S. Non-cementitious Compositions Comprising Vaterite and Methods Thereof. U.S. Patent Application, US201313804558. 2013.3.14.

[4] TAS A. Use of vaterite and calcite in forming calcium phosphate cement scaffolds. Ceram. Eng. Sci. Proc., 2009, 28(9): 135-150.

[5] OHGUSHI H, OKUMURA M, YOSHIKAWA T, et al. Bone formation processin porous calcium carbonate and hydroxyapatite. $J$. Biomed. Mater. Res., 1992, 26(7): 885-895.

[6] BUKREEVA T, MARCHENKO I, BORODINA T, et al. Calcium carbonate and titanium dioxide particles as a basis for container fabrication for brain delivery of compounds. Dokl. Phys. Chem., 2011, 440(1): 165-167.

[7] LI Q, DING Y, LI F, et al. Solvothermal growth of vaterite in the presence of ethylene glycol, 1, 2-propanediol and glycerin. J. Cryst. Growth, 2002, 236(1): 357-362.

[8] FLATEN E, SEIERSTEN M, ANDREASSEN J. Polymorphism and morphology of calcium carbonate precipitated in mixed solvents of ethylene glycol and water. J. Cryst. Growth, 2009, 311(13): 3533-3538

[9] DUPONT L, PORTEMER F. Synthesis and study of a well crystallized $\mathrm{CaCO}_{3}$ vaterite showing a new habitus. J. Mater. Chem., 1997, 7(5): 797-800.

[10] KAMHI S. On the structure of vaterite $\mathrm{CaCO}_{3}$. Acta Crystallogr., 1963, 16(8): 770-772.

[11] MEYER H. Struktur und fehlordnung des vaterits. Z. Kristallogr., 1969, 128(1-6): 183-212.

[12] DEMICHELIS R, RAITERI P, GALE J, et al. A new structural model for disorder in vaterite from first-principles calculations. CrystEngComm, 2012, 14(1): 44-47.

[13] DEMICHELIS R, RAITERI P, GALE J, et al. The multiple structures of vaterite. Cryst. Growth Des., 2013, 13(6): 2247-2251.

[14] NAKA K, TANAKA Y, CHUJO Y. Effect of anionic starburst dendrimers on the crystallization of $\mathrm{CaCO}_{3}$ in aqueous solution: size control of spherical vaterite particles. Langmuir, 2002, 18(9): 3655-3658.

[15] IMAI H, TOCHIMOTO N, NISHINO Y, et al. Oriented nanocrystal mosaic in monodispersed $\mathrm{CaCO}_{3}$ microspheres with functional organic molecules. Cryst. Growth Des., 2012, 12(2): 876-882.

[16] YU S, CÖLFEN H, ANTONIETTI M. Polymer-controlled morphosynthesis and mineralization of metal carbonate superstructures. J. Phys. Chem. B, 2003, 107(30): 7396-7405. 
[17] NEHRKE G, VAN CAPPELLEN P. Framboidal vaterite aggregates and their transformation into calcite: a morphological study. $J$. Cryst. Growth, 2006, 287(2): 528-530.

[18] CÖLFEN H, QI L. A systematic examination of the morphogenesis of calcium carbonate in the presence of a double-hydrophilic block copolymer. Chem.-Eur. J., 2001, 7(1): 106-116.

[19] HU Q, ZHANG J, TENG H, et al. Growth process and crystallographic properties of ammonia-induced vaterite. Am. Mineral., 2012, 97(8/9): 1437-1445.

[20] FRICKE M, VOLKMER D, KRILL C, et al. Vaterite polymorph switching controlled by surface charge density of an amphiphilic dendron-calix [4] arene. Cryst. Growth Des., 2006, 6(5): $1120-1123$.

[21] GEHRKE N, CÖLFEN H, PINNA N, et al. Superstructures of calcium carbonate crystals by oriented attachment. Cryst. Growth Des., 2005, 5(4): 1317-1319.

[22] QI L, LI J, MA J. Biomimetic morphogenesis of calcium carbonate in mixed solutions of surfactants and double-hydrophilic block copolymers. Adv. Mater., 2002, 14(4): 300-303.

[23] MUGNAIOLI E, ANDRUSENKO I, SCHÜLER T, et al. Ab initio structure determination of vaterite by automated electron diffraction. Angew. Chem. Int. Ed., 2012, 51(28): 7041-7045.

[24] WEI H, MA N, SONG B, et al. Formation of multilayered vaterite via phase separation, crystalline transformation, and self-assembly of nanoparticles at the air/water interface. J. Phys. Chem. C, 2007, 111(15): 5628-5632.

[25] WEI H, SHEN Q, ZHAO Y, et al. Influence of polyvinylpyrrolidone on the precipitation of calcium carbonate and on the transformation of vaterite to calcite. J. Cryst. Growth, 2003, 250(3): 516-524.

[26] POLITI Y, ARAD T, KLEIN E, et al. Sea urchin spine calcite forms via a transient amorphous calcium carbonate phase. Science, 2004, 306(5699): 1161-1164.

[27] 章峻, 包富荣, 戴冬萍, 等. 马来酸䣶 (MAH) 表面改性纳米 碳酸钻粉体的制备及表面性能. 无机化学学报, 2007, 23(5): $822-826$.

[28] KONTREC J, KRALJ D, BREČEVIĆ L, et al. Influence of some polysaccharides on the production of calcium carbonate filler particles. J. Cryst. Growth, 2008, 310(21): 4554-4560.

[29] ZHANG Q, REN L, SHENG Y, et al. Control of morphologies and polymorphs of $\mathrm{CaCO}_{3}$ via multi-additives system. Mater. Chem. Phys., 2010, 122(1): 156-163.

[30] 汪小红, 张 群, 董晓庆, 等. 超声辅助的荔枝状球霰石的制备 与表征. 人工晶体学报, 2013, 42(10): 2164-2169.

[31] YANG YA-NAN, ZHU XIAO-LI, KONG XIANG-ZHENG. Con- trols of crystal morphology, size and structure in spontaneous preparation of calcium carbonate. Journal of Inorganic Materials, 2013, 28(12): 1313-1320.

[32] RAMESH T, INCHARA S A, PALLAVI K. Para-amino benzoic acid-mediated synthesis of vaterite phase of calcium carbonate. $J$. Chem. Sci., 2015, 127(5): 843-848.

[33] 夏宏宇, 张 群, 王 刚, 等. 球形和檄榄形球霰石的简易制备研 究. 人工晶体学报, 2015, 44(6): 1701-1706.

[34] ZOU JIAN-PENG, YANG HONG-ZHI, XIAO-PING, et al. Controllable fabrication of calcium carbonate hollow microspheres with micro-nano hierarchical structure. Journal of Inorganic Materials, 2016, 31(7): 711-718.

[35] JI X, LI G, HUANG X. The synthesis of hollow $\mathrm{CaCO}_{3}$ microspheres in mixed solutions of surfactant and polymer. Mater. Lett., 2008, 62(4): 751-754.

[36] 徐国峰, 王洁欣, 沈志刚, 等. 单分散纳米碳酸铨的制备和表征. 北京化工大学学报(自然科学版), 2009, 36(5): 27-30.

[37] KANG S, HIRASAWA I, KIM W, et al. Morphological control of calcium carbonate crystallized in reverse micelle system with anionic surfactants SDS and AOT. J. Colloid. Interf. Sci. 2005, 288(2): 496-502.

[38] JIANG J, MA Y, ZHANG T, et al. Morphology and size control of calcium carbonate crystallized in a reverse micelle system with switchable surfactants. RSC Adv., 2015, 5: 80216-80219.

[39] 陈银霞, 纪献兵, 赵改青, 等. 低温溶剂热法合成圆饼状球霰石 碳酸钙. 材料导报, 2010, 24(12): 99-102.

[40] 陈先勇, 唐 琴, 刘代俊. 独特形貌碳酸钻的微波水热合成与表 征. 功能材料, 2012, 43(9): 1109-1112.

[41] WALSH D, LEBEAU B, MANN S. Morphosynthesis of calcium carbonate (vaterite) microsponges. Adv. Mater., 1999, 11(4): 324-328.

[42] AN X, CAO C. Biomineralization of $\mathrm{CaCO}_{3}$ through the cooperative interactions between multiple additives and self-assembled monolayers. J. Phys. Chem. C, 2008, 112(16): 6526-6530.

[43] YANG B, NAN Z. Abnormal polymorph conversion of calcium carbonate from calcite to vaterite. Mater. Res. Bull., 2012, 47(3): 521-526.

[44] YANG D, YU K, AI Y, et al. The mineralization of electrospun chitosan/poly (vinyl alcohol) nanofibrous membranes. Carbohydr. Polym., 2011, 84(3): 990-996.

[45] RAUTARAY D, AHMAD A, SASTRY M. Biosynthesis of $\mathrm{CaCO}_{3}$ crystals of complex morphology using a fungus and an actinomycete. J. Am. Chem. Soc., 2003, 125(48): 14656-14657.

[46] BEUVIER T, CALVIGNAC B, DELCROIX G, et al. Synthesis of hollow vaterite $\mathrm{CaCO}_{3}$ microspheres in supercritical carbon dioxide medium. J. Mater. Chem., 2011, 21(26): 9757-9761. 
[47] 潘晓芳, 王海水. 乙醇/水混合溶剂体系中碳酸钙晶体晶型和取 向的控制. 无机化学学报, 2014, 30(6): 1312-1316.

[48] 韩金金金, 连 宾, 唐 源, 等. 恶臭假单胞菌对碳酸钙的诱导矿化 作用. 南京大学学报 (自然科学版), 2013, 49(6): 681-688.

[49] 马晓明, 杨媛媛, 张晓婷, 等. 大豆胰岛素指导下具有分级结构 碳酸钻的仿生合成. 河南师范大学学报(自然科学版), 2014, 42(2): 64-68.

[50] 黄玉刚, 禇日环, 何明辉, 等. 富含羧基的多肽基双亲水杂化共 聚物控制碳酸铮的形成. 中山大学学报(自然科学版), 2014, 53(3): 73-79.

[51] GUO Y, WANG F, ZHANG J, et al. Biomimetic synthesis of calcium carbonate with different morphologies under the direction of different amino acids. Res. Chem. Intermed., 2013, 39(6): $2407-2415$.

[52] 任丽英, 张 群, 朱万华, 等. 仿生合成碳酸钻微环. 人工晶体 学报, 2015, 44(1): 250-255.

[53] LIU L, JIANG J, YU S. Polymorph selection and structure evolution of $\mathrm{CaCO}_{3}$ mesocrystals under control of poly (sodium 4-styrenesulfonate): synergetic effect of temperature and mixed solvent. Cryst. Growth Des., 2014, 14(11): 6048-6056.

[54] 陈晓东, 辛梅华, 李明春, 等. N-琥珀酰基-O-羟丙基磺酸壳聚 糖仿生合成球霰石碳酸䥻. 材料研究学报, 2016, 30(1): 31-37.

[55] 朱文杰, 蔡春华, 林嘉平. 碳酸钻在聚合物胶束控制下的仿生 合成. 高分子学报, 2011, 4: 335-339.

[56] 汪玉瑛. 生物成因碳酸钙矿化机制的仿生实验研究. 合肥: 中 国科学技术大学博士学位论文, 2015.

[57] 张 群, 张 清. 不同晶型碳酸钙的仿生矿化研究. 硅酸盐通报, 2014, 33(5): 1236-1240.
[58] JIANG J, YE J, ZHANG G, et al. Polymorph and morphology control of $\mathrm{CaCO}_{3}$ via temperature and PEG during the decomposition of $\mathrm{Ca}\left(\mathrm{HCO}_{3}\right)_{2}$. J. Am. Ceram. Soc., 2012, 95(12): 3735-3738.

[59] JIANG J, ZHANG Y, XU D, et al. Can agitation determine the polymorphs of calcium carbonate during the decomposition of calcium bicarbonate? CrystEngComm, 2014, 16(24): 5221-5226.

[60] ZENG H, YAN Z, JAO M, et al. A novel method for preparing calcium carbonate particles: thermal decomposition from calcium hydrogen carbonate solution. Key Eng. Mater., 2016, 697: 113-118.

[61] TRUSHINA D, BUKREEVA T, KOVALCHUK M, et al. $\mathrm{CaCO}_{3}$ vaterite microparticles for biomedical and personal care applications. Mater. Sci. Eng., C, 2014, 45: 644-658.

[62] RODRIGUEZ-NAVARRO C, JIMENEZ-LOPEZ C, RODRIGUEZ-NAVARRO A, et al. Bacterially mediated mineralization of vaterite. Geochim. Cosmochim. Acta, 2007, 71(5): 1197-1213.

[63] WANG X, WU C, TAO K, et al. Influence of ovalbumin on $\mathrm{CaCO}_{3}$ precipitation during in vitro biomineralization. J. Phys. Chem. B, 2010, 114(16): 5301-5308.

[64] DONNERS J, HEYWOOD B, MEIJER E, et al. Control over calcium carbonate phase formation by dendrimer/surfactant templates. Chem.-Eur. J., 2002, 8(11): 2561-2567.

[65] PARAKHONSKIY B, HAASE A, ANTOLINI R. Sub-micrometer vaterite containers: synthesis, substance loading, and release. Angew. Chem., Int. Edit., 2012, 51(5): 1195-1197.

[66] SAND K, RODRIGUEZ-BLANCO J, MAKOVICKY E, et al. Crystallization of $\mathrm{CaCO}_{3}$ in water-alcohol mixtures: spherulitic growth, polymorph stabilization, and morphology change. Cryst. Growth Des., 2012, 12(2): 842-853. 